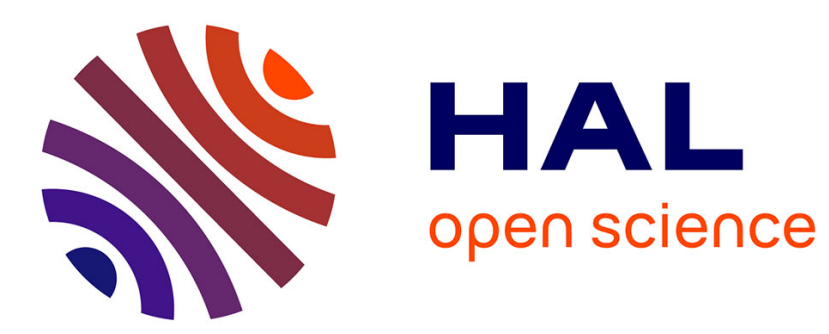

\title{
Corners in phase-field theory
}

Thomas Philippe

\section{To cite this version:}

Thomas Philippe. Corners in phase-field theory. Physical Review E , 2021, 103 (3), 10.1103/Phys-

RevE.103.032801 . hal-03324310

\section{HAL Id: hal-03324310 \\ https://hal.science/hal-03324310}

Submitted on 23 Aug 2021

HAL is a multi-disciplinary open access archive for the deposit and dissemination of scientific research documents, whether they are published or not. The documents may come from teaching and research institutions in France or abroad, or from public or private research centers.
L'archive ouverte pluridisciplinaire HAL, est destinée au dépôt et à la diffusion de documents scientifiques de niveau recherche, publiés ou non, émanant des établissements d'enseignement et de recherche français ou étrangers, des laboratoires publics ou privés. 


\section{Article}

\section{Corners in phase-field theory}

Thomas PHILIPPE

Laboratoire de Physique de la Matière Condensée, Ecole Polytechnique, CNRS, IP Paris, 91128 Palaiseau, France 


\begin{abstract}
Phase field models for strongly anisotropic surface energy need to be regularized to remove the ill-posedness of the dynamic equations. Regularization introduces a new length scale, the corner size, also called bending length. For large corner size, with respect to interface thickness, the phase-field method is known to converge asymptotically toward the sharpinterface theory when the appropriate approximation of the Willmore energy is used. In this work we study the opposite limit, i.e. for corner size smaller than the interface width, and show that the shape of corners, at equilibrium, differs from the sharp-interface picture. However, we find that the phase transition at the interface is preserved and presents the same properties than the classical problem.
\end{abstract}

\title{
Key words
}

phase-field theory, corners, anisotropy, regularization 


\section{Introduction}

Surface energy anisotropy is a predominant factor in interface equilibria. Its effect on the equilibrium crystal shape is a classical problem in material science [1-6]. For strong anisotropy, the equilibrium shape may include sharp corners, even for differentiable surface energy (i.e. no cusps). A corner in an anisotropic crystal spans the so-called missing orientations, i.e. interfacial orientations for which the energy is non-convex. The analogy to a phase transition is due to Cabrera [4]. The equilibrium crystal shape corresponds to the convex part of an appropriate potential, related to the form of the surface energy, and the orientations on both sides of a sharp corner are given by a common tangent condition. In between, the missing orientations may be metastable, or unstable when the stiffness is negative. The later instability is known as spinodal of facets. In case of negative stiffness, however, the dynamic equations are backward parabolic and the mathematical problem is ill-posed and needs to be regularized [4,7].

In the sharp-interface problem, for which the interface is assimilated to a line (in two dimensions), regularization is made by including higher order terms in the surface energy [7-13]. In two dimensions, a curvature-dependent term is added to the dimensionless surface energy $\widetilde{\gamma}$ :

$$
\gamma(\theta)=\widetilde{\gamma}(\theta)+\frac{\alpha^{2}}{2} \kappa^{2}
$$

where $\theta$ is the surface orientation and $\kappa$ the curvature. The curvature-dependent term is known to round the corners over a length $\alpha$ and gives to corners an associated energy. This results in equilibrium shapes with smoothed out corners and circumvents the issue of low wavelength instability in the dynamic setting. Thus, regularization introduces a new length scale, $\alpha$, to the original problem. Spencer [13] showed that the regularized crystal shape converges asymptotically toward the classical shape (with sharp corners) when $\alpha$ tends to zero. This guarantees the validity of the approach, in the sharp-interface framework, when the corner size $\alpha$ is small compared to the other physical lengths of the problem. Spencer [13] also derived the analytical solution for a corner at equilibrium.

On the other hand, interfaces are regarded as being diffuse in many continuum theories. For instance in phase-field theory [14-16], rather than being sharp, the interface has a nonzero thickness over which properties vary smoothly from one set of bulk values to another. The phase-field model has been widely used in material science to model microstructure evolution in many different contexts [17]. It can be viewed as a mathematical tool that allows a reformulation of sharp-interface free-boundary problems [15]. Originally, surface energy anisotropy was introduced in phase-field theories to model dendritic growth [18]. Solidification of metals, one of the main applications of the phase-field method, involves rough interfaces of low anisotropy [19]. However, many other systems including semiconductor materials [20], ceramics or grain boundaries [21], to name a few, may require the description of facets [22], or nearly faceted morphology, for which the surface energy is strongly anisotropic. As sharp-interfaces theories, phase-field models also need to be regularized as the kinetic equations are backward parabolic for negative stiffness. One possibility to 
regularize the model is to convexify the surface energy [23-25] and therefore remove the missing orientations, however, this also suppresses the interfacial phase transition. Wheeler [26] proposed to supplement the standard energy with the square of the Laplacian of the phase-field. This approach preserves the phase transition at the interface, as shown by Wheeler [26], but the model does not converge toward the regularized sharp-interface theory. Significant progress were made by Torabi et al. [27]. In their model, regularization is made using an appropriate approximation of the Willmore energy and the model is constructed such that the interface width is independent on its orientation. It was shown in Refs. [27] and [28] that the model preserves the phase transition at the interface while converging asymptotically toward the regularized sharp-interface theory when the interface thickness, $\varepsilon$, is small compared to the corner size and the other length scales of the problem. As already mentioned the corner size $\alpha$ is also kept small in the sharp-interface theory. Consequently, this phase-field model is a suitable reformulation of the original freeboundary problem in the limit $\varepsilon \ll \alpha \ll 1$.

In the present paper, we investigate the opposite limit, for $\alpha<\varepsilon$. We are interested in deriving the shape of a semi-infinite corner at equilibrium in this regime. To our knowledge, the only solution for a corner in phase-field theory is due to Wheeler [26]. We apply the same methodology. A corner is divided in three different regions, two adjoining interfaces and the corner region, and the method of matched asymptotic expansions is used to find the solution in the different regions. In Sect. 2, we present the phase-field model and perform the asymptotic analysis for a corner in Sect. 3. Wheeler showed that, in the phase-field formulation, the corner problem has the character to a phase transition and is governed by a steady Allen-Cahn equation for the slope. We find a different Allen-Cahn equation and our solution does not converge toward the Spencer calculation [13] for a corner at equilibrium. However, the interfacial phase transition is preserved and presents the same features than the classical problem.

\section{Phase-field model}

We present here the main equations of the regularized phase-field model and gives the asymptotic result for the normal velocity of the interface in the classical limit $\varepsilon \ll \alpha \ll 1$.

\subsection{Governing equations}

The free energy, for the phase-field $u$, is given by [27]:

$$
F=\int f d V
$$

where the energy density $f$ is the the classical phase-field energy to which a regularization term is added:

$$
f=\frac{\Gamma(\vec{n})}{\varepsilon}\left(\Psi(u)+\frac{\varepsilon^{2}}{2}|\vec{\nabla} u|^{2}\right)+\frac{\alpha^{2}}{2 \varepsilon}\left(\frac{\Psi_{u}(u)}{\varepsilon}-\varepsilon \Delta u\right)^{2} .
$$


$\varepsilon$ is the interface width. The last term in Eq. (3) introduces a new length scale, the bending length $\alpha . H \Psi(u)$ is a double-well energy density, here $\Psi(u)=\frac{1}{2}\left(1-u^{2}\right)^{2}$ is dimensionless and the constant $H$ sets the height of the double-well function. $\Psi_{u}$ is the first derivative of $\Psi$ with respect to the phase-field $u . \Gamma(\vec{n})$ is a dimensionless function encoding anisotropy and therefore depends on the orientation of the normal vector $\vec{n}$, defined as: $\vec{n}=\vec{\nabla} u /|\vec{\nabla} u| . \Gamma(\vec{n})$ sets the magnitude of the surface energy $\gamma_{0} \Gamma(\vec{n})$ where $\gamma_{0}$ sets the interfacial energy scale and is related to phase-field parameters by $\gamma_{0}=\widetilde{a} \varepsilon H$ with $\widetilde{a}=\frac{4}{3}$ for our choice of $\Psi(u)$. The regularization term in the energy density is an approximation of the Willmore energy and is due to De Giorgi [29-33].

The Allen-Cahn equation sets the dynamics:

$$
\partial_{t} u=-\frac{1}{\tau^{\prime}} \varepsilon \frac{\delta F}{\delta u}
$$

where

$$
\frac{\delta F}{\delta u}=\frac{1}{\varepsilon}\left(\Gamma(\vec{n}) \Psi_{u}(u)-\varepsilon^{2} \vec{\nabla} \cdot \vec{m}\right)+\frac{\alpha^{2}}{\varepsilon^{3}}\left(\Psi_{u u}(u) w(u)-\varepsilon^{2} \Delta w(u)\right) .
$$

$w(u)=\Psi_{u}(u)-\varepsilon^{2} \Delta u$ and

$$
\vec{m}=\Gamma(\vec{n}) \overrightarrow{\nabla u}+\mathrm{P} \overrightarrow{\nabla_{n}} \Gamma(\vec{n})\left(\frac{\Psi(u)}{\varepsilon^{2}|\vec{\nabla} u|}+\frac{1}{2}|\vec{\nabla} u|\right)
$$

with the projection matrix $\mathrm{P}=\mathrm{I}-\vec{n} \otimes \vec{n}$, I is the identity matrix and $\vec{\nabla}_{n}=\frac{d}{d \vec{n}}$.

Lengths are then rescaled by distance $D$ such as $\widetilde{\varepsilon}=\varepsilon / D \ll 1$ and $\widetilde{\alpha}=\alpha / D \ll 1$. Time is rescaled by $\tau=\tau^{\prime} / \varepsilon^{2}$. $\tau^{\prime}$ is related to the sharp-interface mobility $M$ by $\tau^{\prime}=\varepsilon /(\widetilde{a} H M)$, such that $\tau=D^{2} /\left(M \gamma_{0}\right)$. In two dimensions, the dimensionless evolution equation reads:

$$
\begin{array}{r}
\varepsilon^{4} \partial_{t} u=-\varepsilon^{2}\left(\Gamma(\theta) \Psi_{u}(u)-\varepsilon^{2}\left[\vec{\nabla} \cdot(\Gamma(\theta) \vec{\nabla} u)-\vec{\nabla} \cdot\left(\Gamma_{\theta}(\theta) \vec{\nabla}^{\perp} u\right)\right]\right) \\
+\alpha^{2}\left(\varepsilon^{2} \Delta w(u)-\Psi_{u u}(u) w(u)\right) .
\end{array}
$$

where tildes are omitted for sake of clarity. $\theta$ is the tangent angle to the interface, measured anti-clockwise from the $x$-axis (Fig. 1). $\tan \theta=-n_{x} / n_{y}$ with $n_{x}$ and $n_{y}$ the components of the normal vector $\vec{n}$. Along the front $\zeta$, in the direction of increasing arc-length $s$, the solid domain $\left(\Omega_{+}, u=+1\right)$ lies on the right and liquid $\left(\Omega_{-}, u=-1\right)$ lies on the left. In Eq. (7), $\overrightarrow{\nabla^{\perp}}=\left(\partial_{y},-\partial_{x}\right)$ and the asymptotic result near the interface has been used, $\Psi(u)=\varepsilon^{2}|\vec{\nabla} u|^{2} / 2$. In this approximation, $\vec{m}$ also reads $\vec{m}=-|\vec{\nabla} u| \vec{\Xi}$ with $\vec{\Xi}$ the dimensionless Cahn and Hoffman vector [5], see Appendix A. In two dimensions, $\vec{\Xi}=$ $-\Gamma \vec{n}-\Gamma_{\theta} \vec{t}$ with $-n_{y}$ and $n_{x}$ the components of $\vec{t}$, the tangent vector to the interface. As compared with the classical definition for $\vec{\xi}$, the minus signs come from the fact that with our definition the normal and tangent vectors point in opposite directions. 


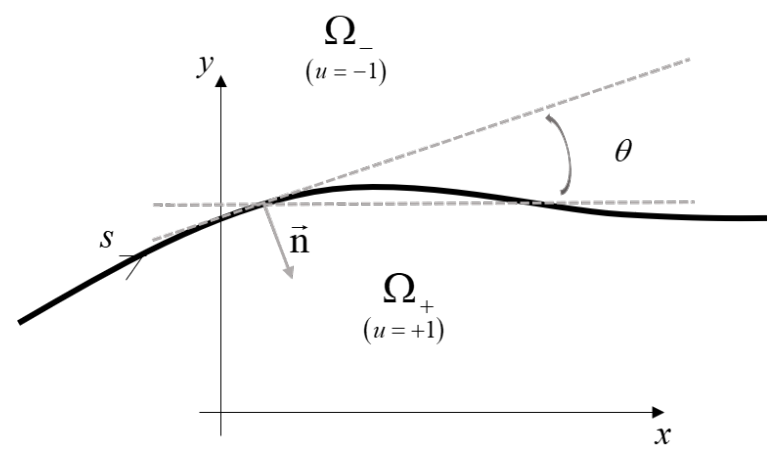

Fig. 1. Schematic of the front $\zeta$.

\subsection{Interface velocity in the classical limit}

The method of matched asymptotic expansions $[29,34,35]$ was used to derive the normal velocity of the interface in the limit $\varepsilon \ll \alpha \ll 1$. In two dimensions, we found [28]:

$$
v_{n}=\widehat{\Gamma}(\theta) \kappa-\alpha^{2}\left(\kappa_{s s}+\frac{1}{2} \kappa^{3}\right)
$$

where $\widehat{\Gamma}(\theta)$ is the dimensionless surface stiffness, $\widehat{\Gamma}(\theta)=\Gamma(\theta)+\Gamma_{\theta \theta}(\theta) . \kappa=\partial_{s} \theta$ is the interface curvature. $\kappa$ is positive when the front $\zeta$ is convex and $v_{n}$ is positive when the front moves toward $\Omega_{-}$, i.e. when crystal grows. Equation (8) is also the dimensionless equation for the sharp-interface velocity [28, 36-39]. Therefore, in the limit $\varepsilon \ll \alpha \ll 1$, the sharp-interface result was recovered. As a consequence, a corner of size $\alpha$ in the phasefield framework is expected to reproduce, at equilibrium $\left(v_{n}=0\right)$, sharp-interface corner's configuration, for which the analytical solution is known [13]. This has been confirmed by phase-field simulations [27]. In the opposite case $\alpha<\varepsilon$, the effect of regularization on corners morphology remains unclear. This regime is investigated in the next section. Our purpose is to determine if the phase transition at the interface is preserved.

\section{Corners}

The sharp-interface problem for regularized corners at equilibrium was solved by Spencer [13]. Using matched asymptotic expansions the explicit solution for a rounded corner was determined. The regularized solution was shown to approach the sharp corner (of the non-regularized equilibrium shape), as the corner size ( $\alpha$ ) approaches zero. The shape of semi-infinite corner was also determined, let us succinctly present this calculation. 


\subsection{Sharp-interface description}

Consider a semi-infinite concave corner $(\kappa<0)$ in equilibrium. Equation. (8), for $v_{n}=0$, can be written as:

$$
\widehat{\Gamma}(\theta) \frac{d \theta}{d s}-\alpha^{2}\left(\frac{d^{3} \theta}{d s^{3}}+\frac{1}{2}\left(\frac{d \theta}{d s}\right)^{3}\right)=0 .
$$

The function $\theta(s)$, that defines the interface profile, satisfies the following boundary conditions: $\theta \rightarrow \theta_{ \pm \infty}$ and $\kappa \rightarrow 0$ as $s \rightarrow \pm \infty$. $\theta_{ \pm \infty}$ are the far-field corner orientations, as given by the common tangent construction on the appropriate surface energy potential: $g=\Gamma / \cos \theta$ [13]. For concave and symmetrical corner, $\theta_{-\infty}=-\theta_{+\infty}>0$ with our definition of $\theta$. Orientations in between are the so-called missing orientations, spanned by the common tangent in the non-regularized equilibrium crystal shape, and are comprised in the nonconvex part of the potential $g$. This analogy with phase transitions was studied by Cabrera and Herring $[2,4]$. The prescription of four boundary conditions to this second order partial differential equation imposes a compatibility condition on the the far-field orientations. Consider the inner length scale $S=\frac{s}{\alpha}$ and $\Theta\left(\frac{s}{\alpha}\right)=\theta(s)$ the arclength parametrization of the scaled profile. The vanishing chemical potential condition (Eq. (9)) becomes:

$$
\left(\partial_{\Theta}^{2}+1\right)\left(\frac{1}{2} \mathfrak{K}^{2}-\Gamma\right)=0
$$

with $\mathfrak{K}=\partial_{S} \theta$. We have $\mathfrak{K}\left(\Theta=\theta_{ \pm \infty}\right)=0$ and $\mathfrak{K}(\Theta) \partial_{\Theta} \mathfrak{K} \rightarrow 0$ as $\Theta \rightarrow \theta_{ \pm \infty}$. Moreover, $\mathfrak{K} \neq 0$ for all $\Theta$ between the far-field orientations. Functions $\phi$ that are multiple of $\cos \Theta$ are solutions of $\left(\partial_{\Theta}^{2}+1\right) \phi=0$. Therefore,

$$
\frac{1}{2} \mathfrak{K}^{2}=\Gamma(\Theta)+A \cos \Theta
$$

with $A$ is a constant determined by the boundary conditions. Substituting its expression in Eq. (11) gives:

$$
\frac{1}{2} \mathfrak{K}^{2}=\Gamma(\Theta)-\Gamma\left(\theta_{ \pm \infty}\right) \frac{\cos (\Theta)}{\cos \left(\theta_{ \pm \infty}\right)} .
$$

Differentiating with respect to $\Theta$ and taking the limit $\Theta \rightarrow \theta_{ \pm \infty}$ yields the common tangent condition:

$$
\partial_{\Theta} \Gamma\left(\theta_{ \pm \infty}\right)+\Gamma\left(\theta_{ \pm \infty}\right) \tan \left(\theta_{ \pm \infty}\right)=0 .
$$

As it must the far-field orientations correspond to the stable orientations from each side of corners of the equilibrium crystal shape. Parametrized curvature $\mathfrak{K}(\Theta)$ is obtained from Eq.(12):

$$
\mathfrak{K}=-\sqrt{2} \sqrt{\Gamma(\Theta)-\Gamma\left(\theta_{-\infty}\right) \frac{\cos (\Theta)}{\cos \left(\theta_{-\infty}\right)}} .
$$

Without loss of generality, the condition $S=0$ in $\Theta=0$ is imposed. Once $\mathfrak{K}$ is known, the profile is constructed using:

$$
S=\int_{0}^{\Theta} \frac{1}{\mathfrak{K}(\mathfrak{u})} d u,
$$


which can be inverted to find $\Theta(S)[13,36,37]$. Eq. (15), together with Eq. (14), gives the shape of a corner in the sharp-interface picture.

\subsection{Diffuse corner theory}

As we mentioned earlier, the present phase-field model converges toward the sharp-interface results, Eq. (8) and Eq. (9), for $\varepsilon \ll \alpha \ll 1$. In this regime, the two regularized models coincide. Therefore, the shape of a corner in phase-field theory shall mimic Eq. (14) and Eq. (15), and this has been confirmed by phase-field simulations [27]. We now investigate the opposite limit, for $\alpha<\varepsilon$. The Allen-Cahn equation, Eq. (7), after rescaling lengths by the dimensionless interface width $\varepsilon$, may be written as

$$
\begin{aligned}
& \varepsilon^{2} \partial_{t} u=-\Gamma(\theta) \Psi_{u}(u)+\left[\vec{\nabla} \cdot(\Gamma(\theta) \vec{\nabla} u)-\vec{\nabla} \cdot\left(\Gamma_{\theta}(\theta) \vec{\nabla}^{\perp} u\right)\right] \\
& +\widehat{\alpha}^{2}\left(\Delta w(u)-\Psi_{u u}(u) w(u)\right) .
\end{aligned}
$$

with $\widehat{\alpha}=\alpha / \varepsilon$. Consider the limit $\varepsilon \rightarrow 0$, followed then by the condition $\widehat{\alpha} \ll 1$. Therefore, we require $\widehat{\alpha} \gg \varepsilon$, i.e. the analysis is made in the particular limit $\varepsilon^{2} \ll \alpha \ll \varepsilon \ll 1$. A corner is pictured Fig. 2. Exterior to each side of the corner, the regions are denoted regions 1 and 2 of Cartesian coordinate systems $\left(r_{1}, q_{1}\right)$ and $\left(r_{2}, q_{2}\right)$, respectively. In the inner corner region, the origin of the coordinate system $(r, q)$, is located in $u=0$ and is common to the coordinate systems of regions 1 and 2 . In this problem there are two interfacial layers, both $u$ and $\vec{\nabla} u$ make a diffuse transition between their bulk values, as we shall demonstrate. For such a corner at equilibrium, the far-field orientations in regions 1 and 2, respectively $\theta_{1}$ and $\theta_{2}$, shall correspond to the thermodynamic orientations, as given by the common tangent of the potential $g$, i.e. the equilibrium values $\theta_{ \pm \infty}$ of the classical force balance at the edge, where the Cahn-Hoffman vectors coincide.

Leading order in $\varepsilon$ gives

$$
\begin{array}{r}
-\Gamma(\theta) \Psi_{u}(u)+\left[\vec{\nabla} \cdot(\Gamma(\theta) \vec{\nabla} u)-\vec{\nabla} \cdot\left(\Gamma_{\theta}(\theta) \vec{\nabla}^{\perp} u\right)\right] \\
+\widehat{\alpha}^{2}\left(\Delta w(u)-\Psi_{u u}(u) w(u)\right)=0 .
\end{array}
$$

In region 1 (and 2, by equivalence) the solution for the phase-field profile is known [28]. Regularization was shown to not modify, in this model, the interfacial profile of planar interfaces in the limit $\varepsilon \ll 1$. Thus,

$$
u\left(r_{1}, q_{1}\right)=\tanh \left(-r_{1}+B_{1} \widehat{\alpha}\right)
$$

with $B_{1}$ a constant to be determined, which represents a translation of the interface. In fact, the two interfaces (regions 1 and 2) adjoining the corner are translated by an extent $\mathscr{O}(\widehat{\alpha})$ with respect to the corner.

Consider now the condition $\widehat{\alpha} \ll 1$. In the thin inner corner region, the lateral coordinate $q$ 


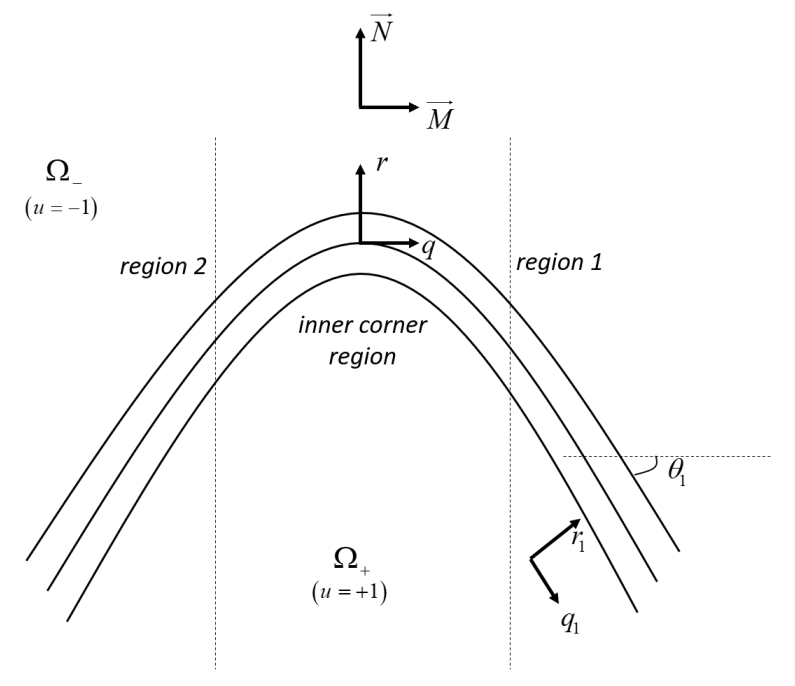

Fig. 2. Schematic of a corner in phase-field theory.

is scaled by $\widehat{\alpha}, l=q / \widehat{\alpha}$. The phase-field in the inner corner region, $U(r, l)$, is expanded in power of $\widehat{\alpha}$ :

$$
U(r, l)=U_{0}(r, l)+\widehat{\alpha} U_{1}(r, l)
$$

In this coordinate system,

$$
\begin{gathered}
\vec{\nabla} u=U_{r} \vec{N}+\frac{U_{l}}{\widehat{\alpha}} \vec{M} \\
\overrightarrow{\nabla^{\perp}} u=-\frac{U_{l}}{\widehat{\alpha}} \vec{N}+U_{r} \vec{M} \\
\Delta u=U_{r r}+\frac{U_{l l}}{\widehat{\alpha}^{2}} \\
\Delta^{2} u=U_{r r r r}+\frac{1}{\widehat{\alpha}^{4}} U_{l l l l}+\frac{2}{\widehat{\alpha}^{2}} U_{r r l l}
\end{gathered}
$$

Substituting those expressions in the Allen-Cahn equation at order $\mathscr{O}(\varepsilon)$, Eq. (17), the leading order problem (at $\mathscr{O}\left(\widehat{\alpha}^{-2}\right)$ ) is

$$
\partial_{l}\left(\Gamma\left(\theta_{0}\right) U_{0, l}\right)-U_{0, l l l l}=0
$$

$\theta_{0}$ is defined from $\vec{\nabla} U$, at leading order. Matching with region 1 (or 2) requires

$$
U_{0}(r)=\tanh \left(-r \cos \left(\theta_{1}\right)\right) .
$$

In the following, we note $\tanh \left(-r \cos \left(\theta_{1}\right)\right)=G(r)$.

The next order, at $\mathscr{O}\left(\widehat{\alpha}^{-1}\right)$, is

$$
\partial_{r}\left(\Gamma_{\theta}\left(\theta_{0}\right) U_{0, l}\right)+\partial_{l}\left(\Gamma\left(\theta_{0}\right) U_{1, l}-\Gamma_{\theta}\left(\theta_{0}\right) U_{0, r}\right)-U_{1, l l l l}=0
$$


Then, we note $v=U_{1, l}$. At leading order,

$$
\vec{\nabla} U^{0}=U_{0, r} \vec{N}+U_{1, l} \vec{M}=G_{r} \vec{N}+v \vec{M}
$$

as $U_{0, l}=0$. Thus, $\tan \left(\theta_{0}\right)=-v / G_{r}$. Eq. (26) may be written as

$$
-v_{l l l}+\partial_{l}\left(\Gamma\left(\theta_{0}\right) v-\Gamma_{\theta}\left(\theta_{0}\right) G_{r}\right)=0
$$

and integrated once with respect to $l$ :

$$
-v_{l l}+\Gamma\left(\theta_{0}\right) v-\Gamma_{\theta}\left(\theta_{0}\right) G_{r}=C
$$

with $C$ a constant. $v_{l l} \rightarrow 0$ as $l \rightarrow \infty$, with concomitantly $\theta_{0} \rightarrow \theta_{1}$, for which the common tangent condition imposes $\Gamma\left(\theta_{1}\right) \tan \left(\theta_{1}\right)+\Gamma_{\theta}\left(\theta_{1}\right)=0$, thus, $C=0$. From $v=$ $-G_{r} \tan \left(\theta_{0}\right)$, we have

$$
v_{l}=-G_{r} \theta_{0, l} \sec ^{2}\left(\theta_{0}\right)
$$

and

$$
v_{l l}=-G_{r}\left(\theta_{0, l l} \frac{1}{\cos ^{2}\left(\theta_{0}\right)}+\left(\theta_{0, l}\right)^{2} 2 \tan \left(\theta_{0}\right)\left(1+\tan ^{2}\left(\theta_{0}\right)\right)\right) .
$$

Hence, Eq. (29) may be written as

$$
\theta_{0, l l} \frac{1}{\cos ^{2}\left(\theta_{0}\right)}+\left(\theta_{0, l}\right)^{2} 2 \tan \left(\theta_{0}\right)\left(1+\tan ^{2}\left(\theta_{0}\right)\right)-\Gamma\left(\theta_{0}\right) \tan \left(\theta_{0}\right)-\Gamma_{\theta}\left(\theta_{0}\right)=0
$$

that gives the orientation profile along the corner, $\theta_{0}(l)$. Introducing the slope $q_{0}=\tan \left(\theta_{0}\right)$, we can deduce an equation for the slope in the inner corner region:

$$
q_{0, l l}=\Gamma\left(q_{0}\right) q_{0}+\Gamma_{q_{0}}\left(q_{0}\right)\left(1+q_{0}^{2}\right)
$$

which can be written as

$$
\Gamma\left(q_{0}\right) q_{0, l l}=\frac{1}{2} \mathscr{G}_{q_{0}}\left(q_{0}\right)
$$

with $\mathscr{G}=g^{2}$. As $g$ is a double-well potential, from which the thermodynamic orientations are derived by construction of the common tangent, the potential $\mathscr{G}$ also is. We find, therefore, a stationary Allen-Cahn equation for the slope, as in Wheeler ([26]), which is characteristic of a phase transition at the interface. This guarantees that the inner edge solution, at leading order, satisfies the common tangent condition, even if the orientation profile is, in this specific regime, quantitatively different from the sharp-interface regularized result, solution of Eq. (9).

Then, $U_{1}$ can be determined from

$$
U_{1}=-G_{r} \int_{0}^{l} q_{0} d l+Q(r)
$$


with $Q(r)$ a yet undetermined function. Therefore, the phase-field solution in the inner edge region is given by

$$
U(r, l)=G(r)+\widehat{\alpha}\left(-G_{r} P(l)+Q(r)\right)
$$

with $P(l)=\int_{0}^{l} q_{0} d l=R(l)+l \tan \left(\theta_{1}\right)$ where $R(l)=\int_{0}^{l}\left(q_{0}-\tan \left(\theta_{1}\right)\right) d l$. Thus,

$$
\begin{array}{r}
U(r, l)=\tanh \left(-r \cos \left(\theta_{1}\right)\right)+ \\
\widehat{\alpha} \tanh ^{\prime}\left(-r \cos \left(\theta_{1}\right)\right)\left(l \sin \left(\theta_{1}\right)+R(l) \cos \left(\theta_{1}\right)+S(r) \cos \left(\theta_{1}\right)\right) .
\end{array}
$$

with $S(r)=-Q(r) / G_{r}$, still undetermined. The phase-field profile in region $1, \tanh \left(-r_{1}+B_{1} \widehat{\alpha}\right)$, can be written, for small $\widehat{\alpha}$, as

$$
U(r, l)=\tanh \left(-r \cos \left(\theta_{1}\right)\right)+\widehat{\alpha} \tanh ^{\prime}\left(-r \cos \left(\theta_{1}\right)\right)\left(l \sin \left(\theta_{1}\right)+B_{1}\right)
$$

since $r_{1}=r \cos \left(\theta_{1}\right)-q \sin \left(\theta_{1}\right)$. Matching the inner corner region $(l \rightarrow \infty)$ with region 1 determines the constants $B_{1}=R(\infty) \cos \left(\theta_{1}\right)$ and $Q(r)=0$. Finally, once the slope profile $q_{0}(l)$ is known, from Eq. (34), the phase-field solution in the inner corner region can be approximated by

$$
U(r, l)=G(r-\widehat{\alpha} P(l))
$$

for $\widehat{\alpha} \ll 1$. This asymptotic solution is compared to a phase field simulation for a corner at equilibrium (for $\widehat{\alpha}=1 / 3$ ), see Fig. (3), and for an arbitrary surface energy, of stable orientations given by the common tangent condition $\pm \pi / 4$. A very good agreement is found. The sharp-interface solution for a corner in equilibrium (Eqs. 14 and 15) is also shown in Fig. (3), for sake of comparison. The slight differences between the sharp-interface solution and the level sets of the asymptotic solution are almost indistinguishable on the scale of the interface width. The asymptotic solution, Eq. (39), is quantitatively different from the corner solution derived by Wheeler [26] since both anisotropy and regularization are implemented in different ways. However, the two solutions are, by nature, very similar. The connection with the wheeler model is made in Appendix A. The interface orientation varies smoothly, within the corner region, through the range of missing orientations and the two interfaces adjoining the corner are translated by an extent $\mathscr{O}(\widehat{\alpha})$. In this regime $(\alpha<\varepsilon)$, the phase transition at the interface is therefore preserved. The interface orientation satisfies the same common tangent construction than the classical problem, even if the corner shape is different from the sharp-interface result. In the opposite limit, $\alpha>\varepsilon$, the present model converges asymptotically toward the sharp-interface theory and, by extension, the corner shape is given by Spencer's calculation, this is not the case for Wheeler's model [26].

\section{Summary}

In deriving the analytical solution for a diffuse corner in the present phase-field model $[27,28]$, we have shown that the phase transition at the interface is also preserved in the 


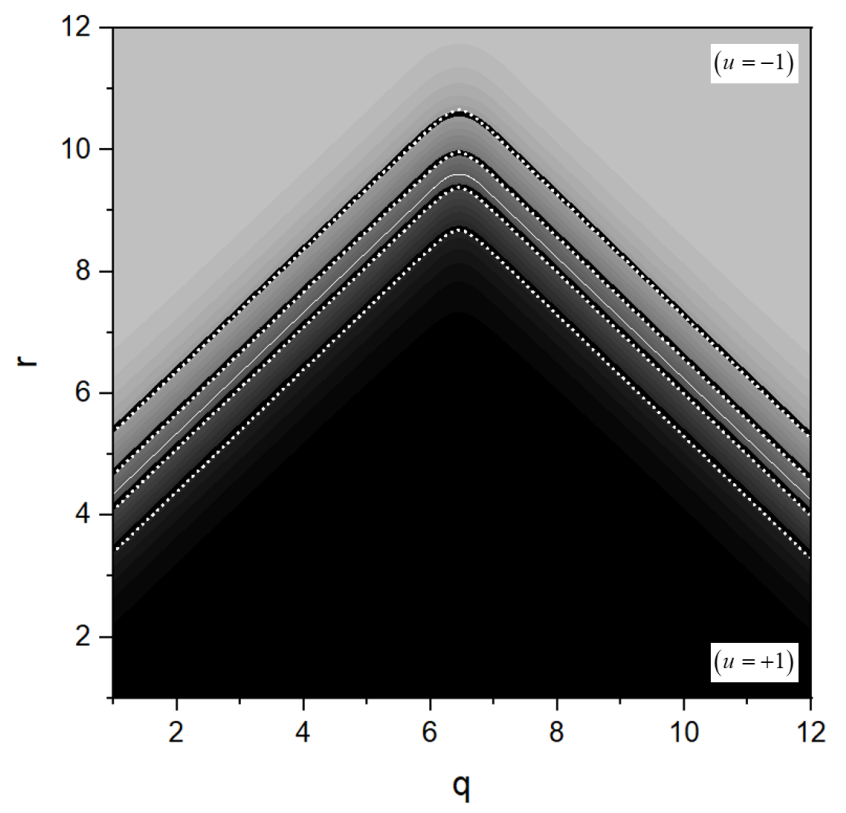

Fig. 3. A corner at equilibrium as given by a phase-field simulation for $\widehat{\alpha}=1 / 3$. Color scale varies from black $(u=+1)$ to grey $(u=-1)$. Level sets of $u$ are represented in black solid lines for the numerical solution and in white dot lines for the asymptotic solution. The solution for a corner in equilibrium in the sharp-interface model is also shown (white solid line) at an arbitrary location (near the level set $u=0$ in this example).

limit $\alpha<\varepsilon$. As in Wheeler [26], the model presents two types of phase transition involving the phase-field $u$ and its gradient $\vec{\nabla} u$. In the region of a diffuse corner, both $u$ and the orientation of the interface, as given by $\vec{\nabla} u$, vary continuously between their equilibrium values. It is found that the interface orientation satisfies the same common tangent construction than the classical problem and varies smoothly through the range of missing orientations in the corner region. As shown by Spencer [13], the regularized models require $\alpha \ll 1$. As a consequence, the classical limit $\varepsilon \ll \alpha$ is rather restrictive in phase-field, from a numerical standpoint. Our calculation justifies larger interface widths, while preserving the interfacial phase transition and its properties, but the asymptotic convergence toward sharp-interface corners is lost.

\section{Acknowledgments}

I acknowledge Mathis Plapp and Hervé Henry (Ecole Polytechnique, France) for fruitful discussions on regularization. 


\section{References}

[1] G. Wulff, Zeitschrift für Kristallographie - Crystalline Materials 34 (1901).

[2] C. Herring, Phys. Rev. 82, 87 (1951).

[3] W. K. Burton, N. Cabrera, and F. C. Frank, Philosophical Transactions of the Royal Society of London. Series A, Mathematical and Physical Sciences 243, 299 (1951).

[4] N. Cabrera, Surface Science 2, 320 (1964).

[5] J. Cahn and D. Hoffman, Acta Metallurgica 22, 1205 (1974).

[6] D. W. Hoffman and J. W. Cahn, Surface Science 31, 368 (1972).

[7] A. Di Carlo, M. E. Gurtin, and P. Podio-Guidugli, SIAM Journal on Applied Mathematics 52, 1111 (1992), https://doi.org/10.1137/0152065 .

[8] J. Stewart and N. Goldenfeld, Phys. Rev. A 46, 6505 (1992).

[9] F. Liu and H. Metiu, Phys. Rev. B 48, 5808 (1993).

[10] A. Golovin, S. Davis, and A. Nepomnyashchy, Physica D: Nonlinear Phenomena 122, 202 (1998).

[11] A. Golovin, S. Davis, and A. Nepomnyashchy, Journal of Crystal Growth 198-199, 1245 (1999).

[12] A. A. Golovin, S. H. Davis, and A. A. Nepomnyashchy, Phys. Rev. E 59, 803 (1999).

[13] B. J. Spencer, Phys. Rev. E 69, 011603 (2004).

[14] J. W. Cahn and J. E. Hilliard, The Journal of Chemical Physics 28, 258 (1958), https://doi.org/10.1063/1.1744102 .

[15] G. Caginalp, in Applications of Field Theory to Statistical Mechanics, edited by L. Garrido (Springer Berlin Heidelberg, Berlin, Heidelberg, 1985) pp. 216-226.

[16] J. S. Langer, "Models of pattern formation in first-order phase transitions," in Directions in Condensed Matter Physics, pp. 165-186, https://www.worldscientific.com/doi/pdf/10.1142/97898144153090005 .

[17] L.-Q. Chen, Annual Review of Materials Research 32, 113 (2002), https://doi.org/10.1146/annurev.matsci.32.112001.132041 .

[18] R. Kobayashi, Physica D: Nonlinear Phenomena 63, 410 (1993).

[19] A. Karma and W.-J. Rappel, Phys. Rev. Lett. 77, 4050 (1996).

[20] F. M. Ross, J. Tersoff, and R. M. Tromp, Phys. Rev. Lett. 80, 984 (1998).

[21] F. Abdeljawad, S. M. Foiles, A. P. Moore, A. R. Hinkle, C. M. Barr, N. M. Heckman, K. Hattar, and B. L. Boyce, Acta Materialia 158, 440 (2018).

[22] E. D. WILliAMS and N. C. BARTELT, Science 251, 393 (1991), https://science.sciencemag.org/content/251/4992/393.full.pdf .

[23] J. Eggleston, G. McFadden, and P. Voorhees, Physica D: Nonlinear Phenomena 150, 91 (2001).

[24] G. Demange, H. Zapolsky, R. Patte, and M. Brunel, npj Computational Materials 3, 15 (2017).

[25] G. Demange, H. Zapolsky, R. Patte, and M. Brunel, Phys. Rev. E 96, 022803 (2017).

[26] A. Wheeler, Proceedings of the Royal Society A: Mathematical, Physical and Engineering Sciences 462, 3363 (2006), 
https://royalsocietypublishing.org/doi/pdf/10.1098/rspa.2006.1721 .

[27] S. Torabi, J. Lowengrub, A. Voigt, and S. Wise, Proceedings of the Royal Society A: Mathematical, Physical and Engineering Sciences 465, 1337 (2009), https://royalsocietypublishing.org/doi/pdf/10.1098/rspa.2008.0385 .

[28] T. Philippe, H. Henry, and M. Plapp, Proceedings of the Royal Society A: Mathematical, Physical and Engineering Sciences 476, 20200227 (2020), https://royalsocietypublishing.org/doi/pdf/10.1098/rspa.2020.0227 .

[29] P. LORETI and R. MARCH, European Journal of Applied Mathematics 11, 203-213 (2000).

[30] Q. Du, C. Liu, and X. Wang, Journal of Computational Physics 198, 450 (2004).

[31] Q. Du, C. Liu, R. Ryham, and X. Wang, Nonlinearity 18, 1249 (2005).

[32] X. Wang, SIAM Journal on Mathematical Analysis 39, 1367 (2008), https://doi.org/10.1137/060663519 .

[33] M. Roger and R. Schatzle, Mathematische Zeitschrift 254, 675 (2006).

[34] A. A. Wheeler, G. B. McFadden, and W. J. Boettinger, Proceedings of the Royal Society of London. Series A: Mathematical, Physical and Engineering Sciences 452, 495 (1996), https://royalsocietypublishing.org/doi/pdf/10.1098/rspa.1996.0026 .

[35] G. B. McFadden, A. A. Wheeler, R. J. Braun, S. R. Coriell, and R. F. Sekerka, Phys. Rev. E 48, 2016 (1993).

[36] S. J. Watson, Proceedings of the Royal Society A: Mathematical, Physical and Engineering Sciences 471, 20140560 (2015), https://royalsocietypublishing.org/doi/pdf/10.1098/rspa.2014.0560 .

[37] T. Philippe, H. Henry, and M. Plapp, Journal of Crystal Growth 503, 20 (2018).

[38] M. E. Gurtin and M. E. Jabbour, Archive for Rational Mechanics and Analysis 163, 171 (2002).

[39] A. DiCarlo, M. E. Gurtin, and P. Podio-Guidugli, SIAM Journal on Mathematical Analysis 52, 1111-1119 (1991).

[40] J. E. Taylor and J. W. Cahn, Physica D: Nonlinear Phenomena 112, 381 (1998).

[41] J.-M. Debierre, A. Karma, F. Celestini, and R. Guérin, Phys. Rev. E 68, 041604 (2003).

[42] P. Bollada, P. Jimack, and A. Mullis, Computational Materials Science 144, 76 (2018).

[43] A. K. Boukellal, A. K. Sidi Elvalli, and J.-M. Debierre, Journal of Crystal Growth 522, 37 (2019).

[44] J. Taylor, J. Cahn, and C. Handwerker, Acta Metallurgica et Materialia 40, 1443 (1992).

\section{Appendix A: The Wheeler model}

Kobayashi [18] was among the firsts to include surface energy anisotropy in phase-field models, in order to simulate dendritic growth. In this class of phase-field models, the 
gradient energy term is dependent on the interface orientation. A similar formulation was studied by Taylor and Cahn [40] in case of faceting. In this context, the energy density reads

$$
f=\frac{1}{\varepsilon}\left(\Psi(u)+\frac{\varepsilon^{2}}{2} \bar{\Gamma}^{2}(\vec{\nabla} u)\right) .
$$

The isotropic case is recovered when $\bar{\Gamma}(\vec{\nabla} u)=|\vec{\nabla} u|$. Surface energy anisotropy is reproduced when $\bar{\Gamma}(\vec{\nabla} u)$ is an appropriate function of $\vec{n}$ [40]. The consequences of nondifferentiability and nonconvexity of gradient energy terms were presented in the Taylor analysis [40]. Nondifferentiability is out of scope of our paper, but the problem was solved by Taylor and Cahn, however, in practice, this solution is almost never used. The usual method consists of regularizing the cusps [41-43]. As it is known, nonconvexity leads to ill-posed equations. Convexification [23, 40] of the surface energy removes the ill-posedness but also the interfacial phase transition. In order to preserve the phase transition Wheeler proposed to regularize in adding the square of the Laplacian of the phase-field to the energy density, Eq. (40). Furthermore, in the Wheeler formulation, the connection with the CahnHoffman $\vec{\xi}$-vector was made in choosing $\bar{\Gamma}(\vec{\nabla} u)=|\vec{\nabla} u| \Gamma(\vec{n})$. This originates from the more general definition of the Cahn-Hoffman vector provided by Taylor [44] where $\bar{\Gamma}(\vec{p})=|\vec{p}| \Gamma\left(\frac{\vec{p}}{|\vec{p}|}\right)$ for all non-zero vectors $\vec{p}$ and where the components of the dimensionless Cahn-Hoffman $\vec{\xi}$-vector are given by $\Xi_{i}=-\frac{\partial \bar{\Gamma}(\vec{p})}{\partial p_{i}}$. The minus sign comes from the fact that, as compared to the classical definition for $\vec{\xi}, \vec{\nabla} u$, and thus the normal vector, point inside the crystal in our model. With those definitions, and adding the regularization term, $\frac{\alpha^{2} \varepsilon}{2}(\Delta u)^{2}$, to the energy density, the dimensionless Allen-Cahn equation can be written as:

$$
\varepsilon^{2} \partial_{t} u=-\Psi_{u}(u)+\vec{\nabla} \cdot[-\bar{\Gamma}(\vec{\nabla} u) \vec{\Xi}(\vec{\nabla} u)]-\widehat{\alpha}^{2} \Delta^{2} u
$$

Lengths have been scaled by the interface thickness. Applying the definition for $\vec{\Xi}$, it can be shown that:

$$
-\bar{\Gamma}(\vec{\nabla} u) \vec{\Xi}(\vec{\nabla} u)=\Gamma^{2}(\vec{n}) \overrightarrow{\nabla u}+|\vec{\nabla} u| \Gamma(\vec{n}) \mathrm{P} \overrightarrow{\nabla_{n}} \Gamma(\vec{n})
$$

where we recognize our definition for $\vec{m}$, up to a factor $\Gamma(\vec{\nabla} u)$, once the asymptotic result near the interface is introduced in the variational expression for $\vec{m}$, Eq. (6). Therefore, the right-hand-side reads $\Gamma(\vec{\nabla} u) \vec{m}$. As the asymptotic result near the interface is used to express $\vec{m}$ in the current formulation of our model, there is, in principle, a first order correction in the corner region since the interfacial profile deviates from the tanh solution. It can be shown that this correction has no effect on the asymptotic analysis, up to first order in $\widehat{\alpha}$. Therefore, our corner solution is also solution of the variational model. Equation (42) shows that the Wheeler model and our phase-field model are connected to the CahnHoffman vector in a similar way, despite implementation of surface energy anisotropy is 
quantitatively different. The other difference is regularization, but our asymptotic analysis in the limit of small corner size (Sec. 3.2) demonstrates that only the square of the Laplacian of the phase-field of Willmore's regularization intervenes in the Allen-Cahn equation, at $\mathscr{O}\left(\widehat{\alpha}^{-2}\right)$ and $\mathscr{O}\left(\widehat{\alpha}^{-1}\right)$, Eq. (24) and Eq. (26). Therefore, up to first order in $\widehat{\alpha}$, effect of regularization is identical in the two phase-field models. As a result, the physics of corners is similar in both models, in the limit of small corner size only. 